Original research article

\title{
Acute effect of spinal cord stimulation on autonomic nervous system function in patients with heart failure
}

\author{
Jan Naar ${ }^{1 *}$, Deborah Jaye ${ }^{2}$, Petr Neužil ${ }^{1}$, Petr Doškář ${ }^{1}$, Filip Málek ${ }^{1}$, Bengt Linderoth ${ }^{3}$, \\ Göran Lind ${ }^{3}$, Marcus Ståhlberg ${ }^{3}$ \\ ${ }^{1}$ Na Homolce Hospital, Department of Cardiology, Prague, Czech Republic \\ ${ }^{2}$ Medtronic Plc, Cardiac Rhythm and Heart Failure, Minneapolis, USA \\ ${ }^{3}$ Karolinska Institutet and Karolinska University Hospital, Stockholm, Sweden
}

\begin{abstract}
Aims: To test the hypothesis that spinal cord stimulation (SCS) acutely improves heart rate variability (HRV) and baroreceptor sensitivity (BRS) in patients with heart failure (HF).

Methods: SCS (15 minutes) was delivered in four different settings: 90\% of maximal tolerated stimulation amplitude (MTA) targeting the T1-T4 spinal cord segments (SCS90T1-4), 60\% of MTA (SCS60T1-4), 90\% of MTA with cranial (SCS90CR) and caudal (SCS90CA) electrode configuration. HRV and BRS were recorded continuously and stimulation was compared to device off.

Results: Fifteen HF patients were included. SCS90T1-4 did not change the standard deviation of intervals between normal beats (SDNN, $p=0.90)$, BRS $(p=0.55)$ or other HRV parameters. In patients with baseline SDNN <50 ms, SCS90T1-4 significantly increased SDNN $(p=0.004)$.

Conclusions: Acute SCS at $60-90 \%$ of MTA targeting upper thoracic spinal cord segments does not improve autonomic balance or baroreceptor sensitivity in unselected patients with heart failure but may improve HRV in patients with low SDNN.
\end{abstract}

Keywords: Baroreceptor sensitivity; Heart failure; Heart rate variability; Spinal cord stimulation

Highlights:

- SCS at T1-T4 segments did not acutely improve HRV in unselected patients with HF.

- SCS acutely improved HRV in HF patients with low baseline HRV.

- Baseline autonomic function may influence the response to SCS therapy in HF patients.

- This should be taken into account during recruitment for neuromodulation trials.

\section{Introduction}

Heart failure is characterized by sympathetic nervous system overactivity and vagal withdrawal (Azevedo and Parker, 1999; Parker, 1992). This autonomic imbalance has deleterious longterm effects and is accompanied by attenuated arterial baroreflex control, e.g. blunted baroreceptor sensitivity (BRS) (Wang et al., 1990). Increased sympathetic drive (Jacobson et al., 2010; Nakata et al., 2013) and blunted BRS (Mortara et al., 1997; Osterziel et al., 1995) are independent predictors of adverse outcome in heart failure.

Spinal cord stimulation (SCS) is a treatment option for adults with chronic intractable pain of neuropathic or ischemic origin, including refractory angina pectoris (Simpson et al., 2009). In canine models, SCS applied to the upper thoracic segments (T1-4) of the spinal cord elicits a direct sympatholytic effect on the heart by modulating efferent cardiac neu- ronal signalling (Foreman et al., 2000). In experimental heart failure models, the sympatholytic effect of SCS translates into improved left ventricular function and volumes and reduced propensity for ventricular arrhythmias (Lopshire et al., 2009). Importantly, stimulation amplitude and targeted thoracic spinal cord segments both modulated the sympatholytic effect of SCS (Lopshire and Zipes, 2014).

Heart rate variability (HRV) is thought to reflect autonomic function and its evaluation is usually automated using one of many commercial systems. This enables assessment of the key pathophysiological change in heart failure: impaired regulation of the cardiac autonomic nervous system. Likewise, BRS is an established tool for the assessment of autonomic control of the cardiovascular system and can also be quantified non-invasively. Thus, change in baroreflex function reveals alteration in autonomic control of the cardiovascular system (La Rovere et al., 2008). It has previously been shown that HRV improves with SCS in patients with refractory angina pectoris without

\footnotetext{
* Corresponding author: Jan Naar, Na Homolce Hospital, Department of Cardiology, 15003 Prague 5, Czech Republic; e-mail: jan.naar@seznam.cz http://doi.org/10.32725/jab.2021.012

Submitted: 2020-10-08 • Accepted: 2021-05-05 • Prepublished online: 2021-05-18

J Appl Biomed 19/3: 133-141 • EISSN 1214-0287 • ISSN 1214-021X

(c) 2021 The Authors. Published by University of South Bohemia in České Budějovice, Faculty of Health and Social Sciences.

This is an open access article under the CC BY-NC-ND license.
} 
heart failure (Anselmino et al., 2009; Moore et al., 2004). However, the acute effect of SCS on HRV and BRS in heart failure subjects remains largely unknown.

Therefore, the main aim of this study was to test the hypothesis that SCS acutely improves HRV and BRS in patients with heart failure. A secondary objective was to examine the impact of different stimulation amplitudes and targeted thoracic spinal cord segments on the effect of SCS.

\section{Materials and methods}

\section{Patient selection}

Patients participating in the DEFEAT-HF clinical trial were recruited from two study centers (Karolinska University Hospital, Stockholm, Sweden and Na Homolce Hospital, Prague, Czech Republic). The DEFEAT-HF trial was designed to evaluate the effect of SCS on left ventricular remodeling in patients with heart failure and reduced ejection fraction (Zipes et al., 2016). Main inclusion criteria in the DEFEAT-HF trial were age $\geq 18$ years, New York Heart Association functional class (NYHA) III, left ventricular ejection fraction $\leq 35 \%$, QRS duration $<120 \mathrm{~ms}$ and left ventricular end-diastolic diameter of $55-80 \mathrm{~mm}$. Main exclusion criteria in the DEFEAT-HF trial were coronary artery revascularization or acute coronary syndrome within 90 days of enrolment, a reversible type of left ventricular systolic dysfunction, cardiac resynchronization therapy and severe mitral regurgitation. In the present study, we included participants from the DEFEAT-HF study with $\geq 6$ months SCS therapy and excluded patients that were unwilling or unable to comply with study procedures.

\section{Spinal cord stimulation}

The SCS device consists of a single octopolar lead (model 3777/3877, Medtronic, Plc) implanted in the epidural space, targeting the midline of the $\mathrm{T} 1-\mathrm{T} 4$ segments of the spinal cord. The lead is connected with a PrimeADVANCED ${ }^{\mathrm{TM}}$ pulse generator (Model 37702, Medtronic, Plc) that is placed subcutaneously in the region of the lateral abdomen. A stimulation frequency of $50 \mathrm{~Hz}$ and pulse duration of $210 \mu$ s were used.

\section{Study protocol}

Fig. 1A shows an outline of the study protocol. Data were collected during an outpatient visit 6 weeks after discontinuation of SCS therapy (wash-out). Study visits for all patients were conducted at approximately the same time of day and the patients were instructed to abstain from food and beverages containing caffeine or alcohol $>12$ hours before the investigation. None of the patients were on hormone replacement therapy.

First, sensitivity testing to determine maximal tolerated amplitude (MTA) and dermatome mapping were performed. Patients then rested comfortably in a supine position for $>15$ minutes before any measurements or interventions were started. Subsequently, four SCS settings were programmed for 15 minutes each in sequential order (Fig. 1B): 90\% of MTA targeting the T1-T4 spinal cord segments (SCS90T1-4), 60\% of MTA in the T1-T4 segments (SCS60T1-4) and 90\% of MTA with cranial (SCS90CR) and caudal (SCS90CA) electrode configuration. We applied a 35-minute period of SCS off between each intervention to allow for wash-out of the previous stimulation. Throughout the protocol, continuous electrocardiogram (ECG) and beat-to-beat hemodynamic data using a non-invasive hemodynamic monitor Nexfin ${ }^{\circledR}$ (BMEYE, Amsterdam, Netherlands) were collected. Cardiac output assessed by a foreign gas rebreathing device (Innocor ${ }^{\circledR}$, Innovision, Odense, Denmark) (Agostini and Cattadori, 2009) was used to calibrate Nexfin cardiac output measures. No change in medical therapy was allowed during the protocol.

Local authorities and Ethics Committees at both participating centers approved the study protocol and the study protocol was in compliance with the principles of the Declaration of Helsinki. All subjects provided written informed consent.

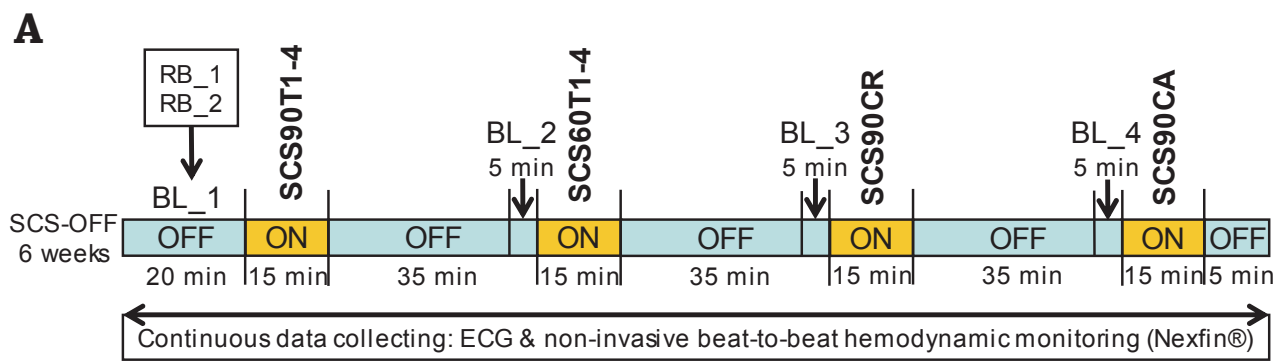

B

SCS90T1-4
SCS60T1-4
SCS90CR
SCS90CA

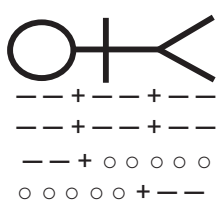

$90 \%$ of maximal tolerable amplitude $60 \%$ of maximal tolerable amplitude $90 \%$ of maximal tolerable amplitude $90 \%$ of maximal tolerable amplitude

Fig. 1. Outline of the study protocol (A). Spinal cord electrode configurations and outputs used during intervention periods throughout the protocol (B). BL - baseline; ECG - electrocardiogram; OFF - spinal cord stimulation inactive; ON - spinal cord stimulation active; RB - inert gas rebreathing test; SCS - spinal cord stimulation; SCS60T1-4 - spinal cord stimulation with the amplitude of $60 \%$ of maximal tolerated amplitude targeting T1-T4 spinal cord segments; SCS90T1-4 - spinal cord stimulation with the amplitude of $90 \%$ of maximal tolerated amplitude targeting T1-T4 spinal cord segments; SCS90CA - spinal cord stimulation with the amplitude of 90\% of maximal tolerated amplitude and an electrode configuration targeting caudal segments; SCS90CR - spinal cord stimulation with the amplitude of $90 \%$ of maximal tolerated amplitude and an electrode configuration targeting cranial segments. 


\section{Assessment of heart rate variability}

Analysis was performed using PowerLab and LabChart software (ADInstruments, Dunedin, New Zealand). ECG recordings of the last five minutes of each intervention and the last five minutes of each preceding SCS-OFF period were used to analyze HRV according to recommendations from the European Society of Cardiology Task Force (Heart rate variability..., 1996). In the present study, we used three time-domain measures: standard deviation of intervals between normal beats (SDNN), square root of the mean of the sum of the squares of differences between adjacent NN intervals (RMSSD), number of pairs of adjacent NN intervals differing by more than $50 \mathrm{~ms}$ divided by the total number of all NN intervals (pNN50), and several frequency-domain methods: high frequency (HF: $0.15-0.40 \mathrm{~Hz}$ ) reflecting vagal activity, low frequency (LF: $0.04-0.15 \mathrm{~Hz}$ ) reflecting baroreflex modulation of autonomic function (Goldstein et al., 2011; Rahman et al., 2011), very low frequency (VLF: $<0.04 \mathrm{~Hz}$ ) representing the strongest prognostic marker from all frequency bands (Hadase et al., 2004) and LF to HF ratio (LF/HF). Frequency-domain measures are expressed as power in each frequency range $\left(\mathrm{ms}^{2}\right)$. Previous studies have shown that SDNN $<50 \mathrm{~ms}$ confers a particularly poor prognosis and this cut-off is recommended by the European Society of Cardiology Task Force for prognostication (Heart rate variability..., 1996). Therefore, we also tested the effect of SCS separately in patients with baseline SDNN $>50 \mathrm{~ms}$ versus $<50 \mathrm{~ms}$.

\section{Assessment of baroreceptor sensitivity}

Spontaneous BRS was estimated by the sequence method as described previously (Parlow et al., 1995; Wang et al., 2004). Beat-to-beat systolic blood pressure (sBP) and RR intervals were analyzed using a custom MATLAB algorithm selecting all sequences of three or more successive beats where there were concomitant increases or decreases in SBP and RR interval. The average regression slope was calculated in different 5-minute segments for each stimulation setting (0-5 minute, 5-10 minute, last 5 minutes if total stimulation period was $\geq 12.5$ minutes) and compared with baseline (5-minute segment preceding the stimulation period). The regression slope (expressed as $\mathrm{ms} / \mathrm{mmHg}$ ) is a representative of spontaneous BRS.

\section{Assessment of hemodynamic data}

Heart rate, sBP, diastolic blood pressure (dBP), mean arterial pressure (MAP), stroke volume (SV) and cardiac output were measured. An estimate of total peripheral resistance (eTPR) was calculated as: $\mathrm{MAP} / \mathrm{CO} \times 79.9\left(\mathrm{dyn} / \mathrm{s} / \mathrm{cm}^{-5}\right)$. A five-minute segment during SCS-OFF preceding each stimulation setting and 15-minute stimulation periods were used for hemodynamic analyses.

\section{Statistical analysis}

Normal distribution of HRV, BRS and hemodynamic data was controlled with the Shapiro-Wilk normality test, and parametric (paired two-tailed $t$-test) and non-parametric (Wilcoxon two-tailed matched pairs test) testing was used as appropriate. Data are presented as mean with $95 \%$ confidence interval (CI) for normally distributed data and median with $95 \%$ CI for skewed data. Differences between low and high baseline SDNN subgroups were compared using an unpaired two-tailed $t$-test. All statistical analyses were performed using GraphPad Prism version 8.3.0 (GraphPad Software, La Jolla California, USA). $P$-values $<0.05$ were considered statistically significant.

\section{Results}

\section{Baseline characteristics and therapy delivery}

Table 1 shows the baseline characteristics of the study population. Fifteen subjects ( 4 female, age $67 \pm 8$ years, $53 \%$ ischemic heart failure etiology) were enrolled. Average left ventricular ejection fraction and indexed end-diastolic volume (LVEDV) were $41 \pm 14 \%$ and $162 \pm 48 \mathrm{ml} / \mathrm{m}^{2}$, respectively. Average NYHA class was $2.6 \pm 0.6$.

Table 1. Baseline characteristics

\begin{tabular}{lc}
\hline Age (years) & $67 \pm 8$ \\
Female sex ( $n$; \%) & $4(26)$ \\
NYHA class & $2.6 \pm 0.6$ \\
LVEF (\%) & $41 \pm 14$ \\
LVEDV (ml) & $162 \pm 48$ \\
Systolic blood pressure (mmHg) & $124 \pm 18$ \\
Diastolic blood pressure (mmHg) & $74 \pm 9$ \\
BMI (kg/m ${ }^{2}$ ) & $28 \pm 6$ \\
Heart failure duration (years) & $4.3 \pm 2.3$ \\
Ischemic etiology ( $n$; \%) & $8(53)$ \\
Previous CABG/PCI $(n ; \%)$ & $6(40)$ \\
History of hypertension $(n ;$ \%) & $10(67)$ \\
COPD ( $n$; \%) & $2(13)$ \\
Current or past smoker $(n ; \%)$ & $9(60)$ \\
Diabetes mellitus $(n ; \%)$ & $2(13)$ \\
ICD implanted $(n ; \%)$ & $13(87)$ \\
$\%$ of patients on $\beta$-blocker/ACEI or ARB/MRA & $100 / 93 / 87$ \\
$\%$ of target daily dose of $\beta$-blocker & 66 \\
$\%$ of target daily dose of ACEI/ARB & 64 \\
\hline
\end{tabular}

ACEI - angiotensin-converting enzyme inhibitor; $\mathrm{ARB}$ - angiotensin II receptor blocker; BMI - body mass index; $C A B G$ - coronary artery bypass grafting; COPD - chronic obstructive pulmonary disease; ICD - implantable cardioverter defibrillator; LVEF - left ventricular ejection fraction; LVEDV - left ventricular end-diastolic volume; MRA - mineralocorticoid receptor antagonist; NYHA class - New York Heart Association functional class; PCI - percutaneous coronary intervention.

MTA was $3.3 \pm 2.9 \mathrm{~V}, 3.5 \pm 2.9 \mathrm{~V}$ and $4.2 \pm 2.7 \mathrm{~V}$ respectively in SCS90T1-4, SCS90CR and SCS90CA. Distribution of perceived paresthesia during dermatome mapping in the three SCS lead configurations is displayed in Suppl. Figs. S1-S3.

\section{Acute effect of SCS on heart rate variability}

As shown in Fig. 2, heart rate was unchanged when comparing SCS90T1-4 (63.2 [49.4-76.8] bpm) to SCS-OFF (65.6 [53.575.3] bpm, $p=0.29$ ), and SDNN did not change significantly with SCS90T1-4 compared to SCS-OFF (53.0 [27.3-78.1] ms versus 55.7 [22.8-105.3] $\mathrm{ms}, p=0.90)$. No statistically significant differences in heart rate or SDNN were observed with SCS60T1-4, SCS90CR or SCS90CA. Similarly, we did not prove any change in RMSSD or pNN50 with SCS compared to SCSOFF (Suppl. Fig. S4). As shown in Fig. 3, there was no significant change in any frequency-domain HRV parameter with SCS compared to SCS-OFF regardless of stimulation amplitude or lead configuration. 


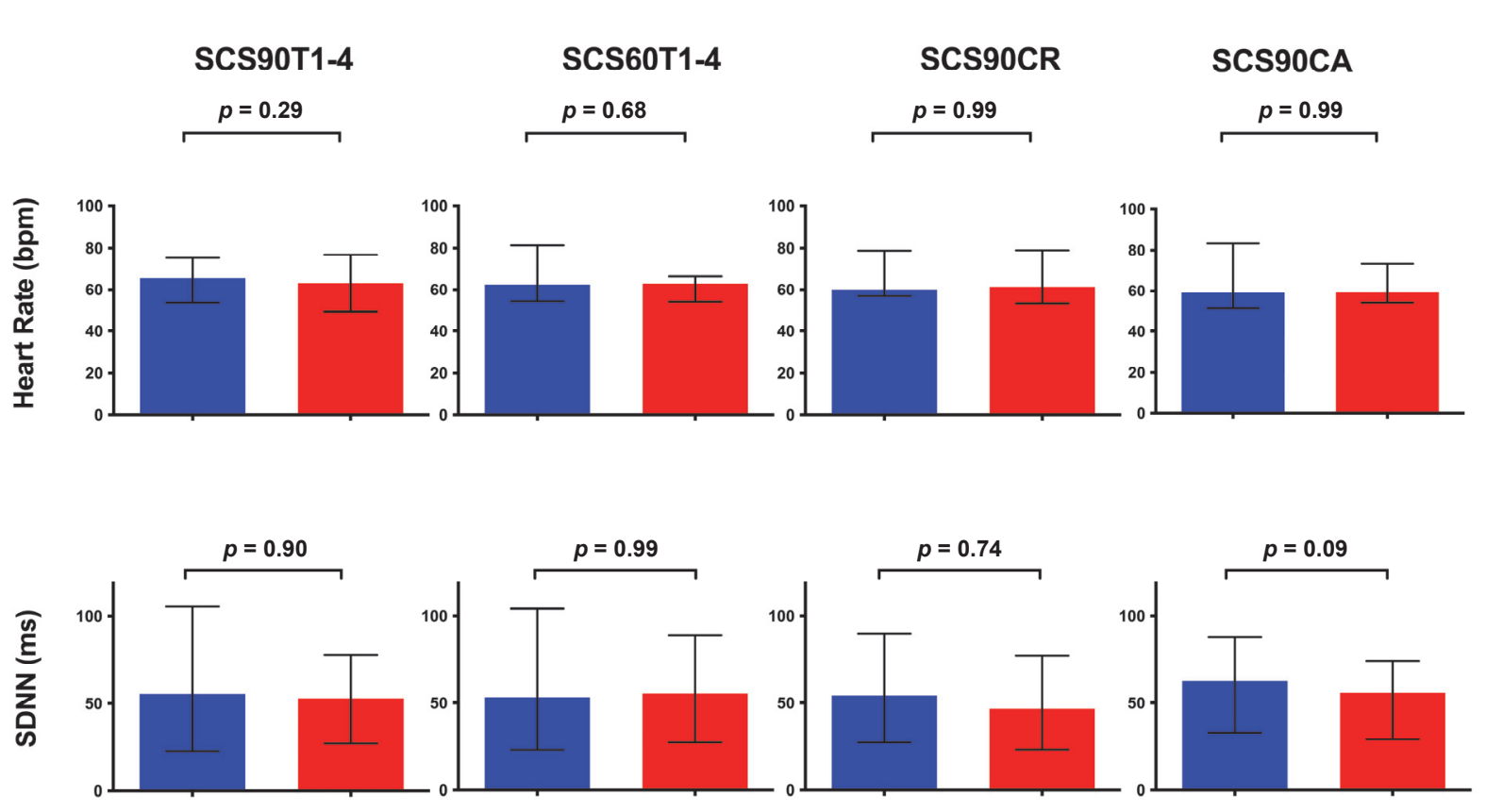

SCS-OFF

SCS-ON

Fig. 2. Acute effect of SCS with different stimulation amplitudes and electrode configurations on heart rate and SDNN. Data are expressed as median with $95 \%$ confidence interval. Bpm - beats per minute; SDNN - standard deviation of intervals between normal beats.
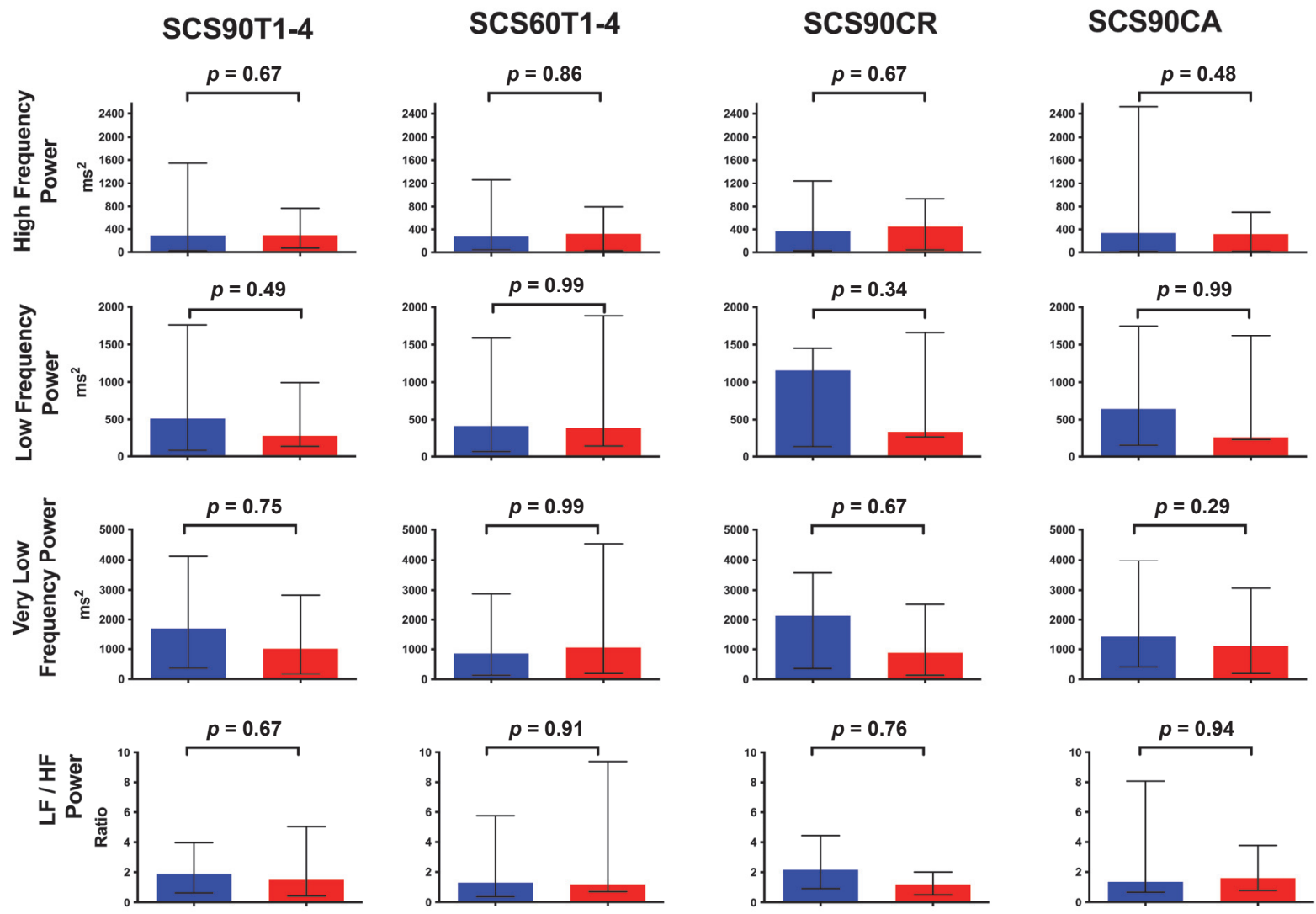

Fig. 3. Acute effect of SCS with different stimulation amplitudes and lead configurations on frequency-domain heart rate variability parameters. Data are expressed as median with $95 \%$ confidence interval. 


\section{Effect of baseline SDNN on SCS therapy}

In the subgroup analysis, patients with low baseline SDNN ( $<50 \mathrm{~ms}, n=4$ ) displayed significantly increased SDNN with SCS90T1-4 compared to SCS-OFF, whereas SDNN in patients with high baseline SDNN ( $>50 \mathrm{~ms}, n=7$ ) did not change significantly with SCS (Fig. 4). This pattern was not observed with lower stimulation amplitude or other electrode configurations, where SDNN did not change with SCS (Suppl. Fig. S5). Table 2 shows the baseline characteristics of low and high baseline SDNN subgroups. Although the patients with baseline SDNN $<50 \mathrm{~ms}$ tended to be more symptomatic and have lower ejection fraction, more pronounced left ventricular dilation and less titrated pharmacotherapy of heart failure, the low number of participants in subgroups does not permit correct statistical analysis.
A

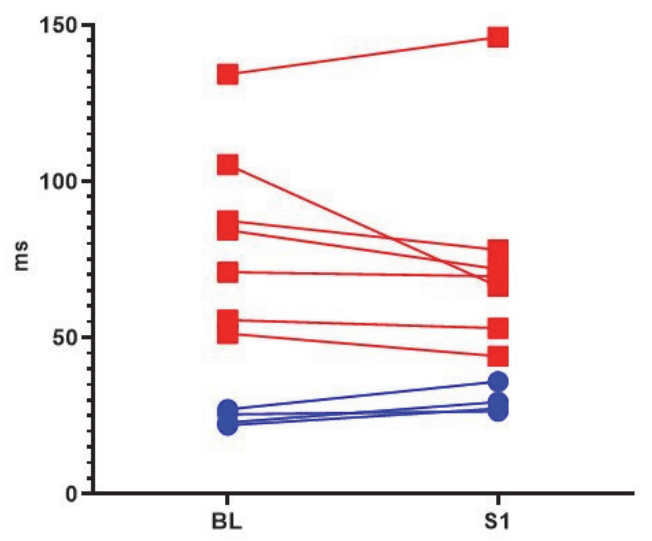

Baseline SDNN >50

Baseline SDNN $<50$
Ratio SDNN change B SCS90T1-4 vs. SCS-OFF

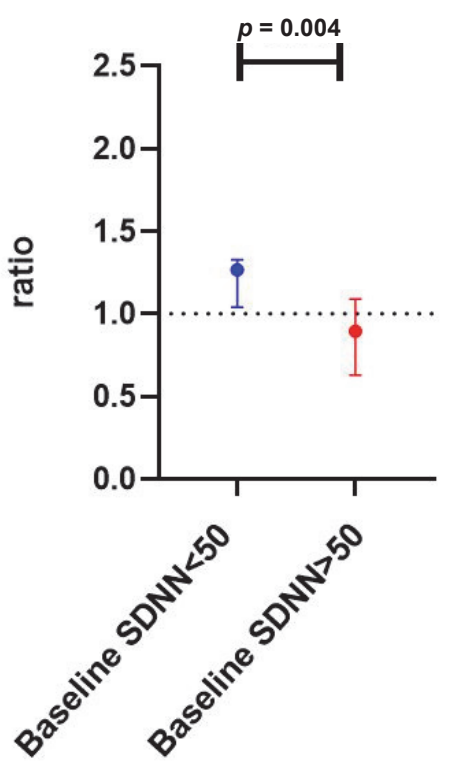

Fig. 4. Acute effect of SCS with $90 \%$ of maximal tolerated amplitude on SDNN in patients with low ( $<50 \mathrm{~ms})$ versus high ( $>50 \mathrm{~ms})$ baseline SDNN. Data are expressed as individual (A) and median with $95 \%$ confidence interval of delta ratios (B). SDNN - standard deviation of intervals between normal beats.

Table 2. Baseline characteristics of low and high SDNN subgroups

\begin{tabular}{|c|c|c|c|}
\hline & $\begin{array}{c}\text { SDNN }<50 \mathrm{~ms} \\
(n=4)\end{array}$ & $\begin{array}{c}\text { SDNN }>50 \mathrm{~ms} \\
(n=7)\end{array}$ & $p$-value \\
\hline Age (years) & $64 \pm 10$ & $68 \pm 9$ & 0.5 \\
\hline BMI $\left(\mathrm{kg} / \mathrm{m}^{2}\right)$ & $29.6 \pm 7$ & $27.2 \pm 7$ & 0.6 \\
\hline Systolic blood pressure, $\mathrm{mmHg}$ & $130 \pm 21$ & $122 \pm 20$ & 0.53 \\
\hline Diastolic blood pressure, $\mathrm{mmHg}$ & $78 \pm 9$ & $70 \pm 9$ & 0.24 \\
\hline NYHA class & $2.8 \pm 0.5$ & $2.3 \pm 0.5$ & 0.17 \\
\hline $\operatorname{LVEF}(\%)$ & $34 \pm 11$ & $44 \pm 15$ & 0.27 \\
\hline $\operatorname{LVEDV}(\mathrm{ml})$ & $170 \pm 62$ & $139 \pm 15$ & 0.26 \\
\hline Time since heart failure diagnosis (years) & $3.3 \pm 1$ & $4 \pm 2$ & 0.55 \\
\hline Ischemic etiology ( $n ; \%)$ & $1(25 \%)$ & $5(71 \%)$ & \\
\hline$\%$ of target daily dose of $\beta$-blocker & $47 \pm 39$ & $82 \pm 43$ & 0.21 \\
\hline$\%$ of target daily dose of ACEI/ARB & $32 \pm 21$ & $80 \pm 35$ & 0.04 \\
\hline
\end{tabular}

ACEI - angiotensin-converting enzyme inhibitor; ARB - angiotensin II receptor blocker; BMI - body mass index; LVEF - left ventricular ejection fraction; LVEDV - left ventricular end-diastolic volume; NYHA class - New York Heart Association functional class; SDNN - standard deviation of intervals between normal beats. 


\section{Acute effect of SCS on baroreceptor sensitivity}

We did not observe any significant acute effect of SCS on BRS regardless of amplitude and lead configuration tested (Fig. 5).

\section{Acute effect of SCS on hemodynamic parameters}

As detailed in Fig. 6, we did not observe any significant change in hemodynamic parameters with other SCS configurations except for a significant increase in sBP and dBP with SCS60T1-4 (118 \pm 15 versus $115 \pm 16 \mathrm{mmHg}, p=0.03$ and $65 \pm 6$ versus $63 \pm 7 \mathrm{mmHg}, p=0.04)$.
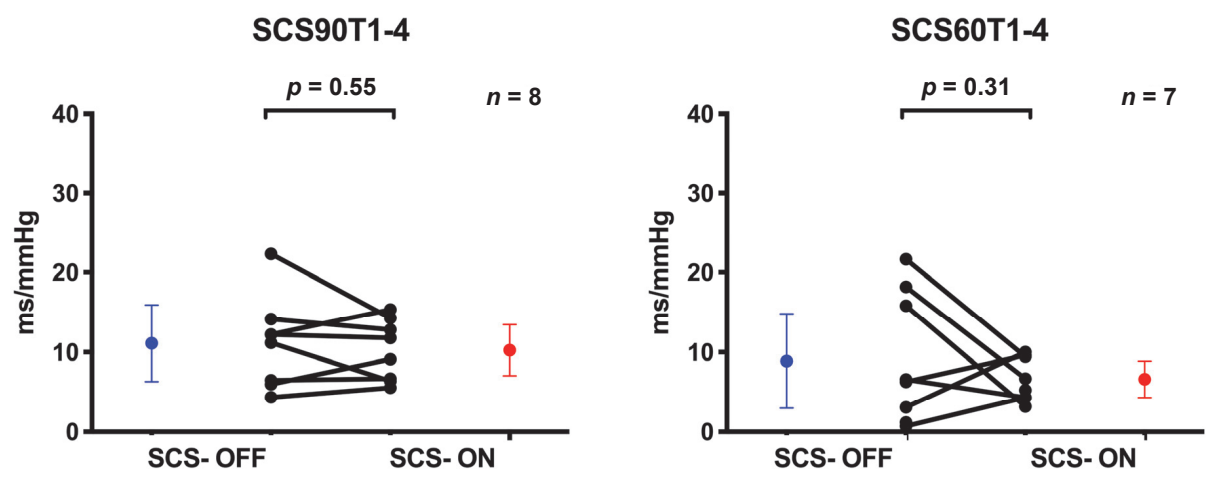

\section{SCS90CR}

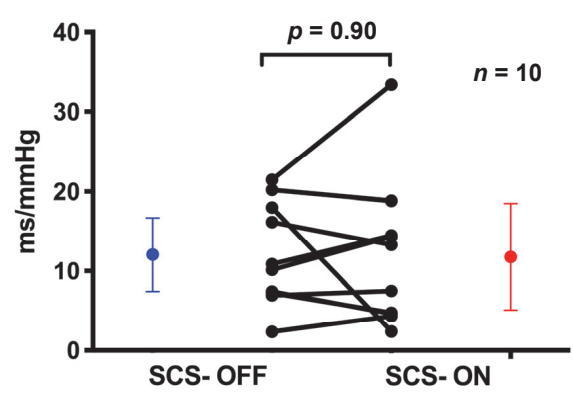

SCS90CA

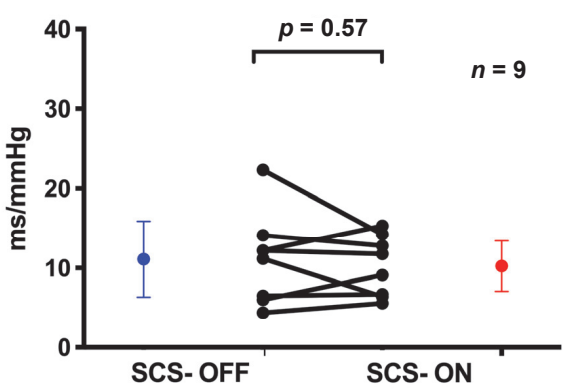

Fig. 5. Acute effect of SCS with different amplitudes and lead configurations on baroreceptor sensitivity. Results are displayed as individual, as well as median with $95 \%$ confidence interval.

\section{Discussion}

This is the first clinical study investigating the acute effect of SCS on HRV and baroreceptor reflex control in patients with heart failure. We did not detect any acute salutary effects in the unselected sample of patients. However, we observed an association between the HRV at baseline and a significant increase in SDNN with SCS, indicating a favourable effect of SCS on the autonomic function in patients with an initially low HRV.

\section{Effect of SCS on the autonomic nervous system in subjects without heart failure}

There is preclinical and clinical trial evidence that SCS improves autonomic nervous system balance in subjects without heart failure. Foreman et al. (2000) showed that SCS suppresses cardiac sympathetic nerve activity evaluated by direct microneurography in anesthetized dogs with and without myocardial ischemia. SCS has also been shown to reduce susceptibility to atrial fibrillation in non-failing canine models (Bernstein et al., 2012). Furthermore, two clinical studies have documented an acute effect of SCS on HRV in patients with refractory angina but without heart failure. Moore et al. (2004) observed significant reductions in LF/HF and LF in a study with sixteen subjects suffering from refractory angina pectoris, and Anselmino et al. (2009) documented a significant reduction of LF/HF in eight patients with refractory angina.

However, it is not trivial to extrapolate these data to a heart failure population due to intrinsic differences in autonomic balance and reflex regulation between patients with and without heart failure.

\section{Effect of SCS on the autonomic nervous system in subjects with heart failure}

Several preclinical studies on ischemia/reperfusion and myocardial infarction (but not heart failure per se) models have shown that SCS reduces infarct size, prevents ventricular arrhythmias and improves stressor tolerance (Cardinal et al., 2004; Issa et al., 2005; Odenstedt et al., 2014). Preclinical data of SCS efficacy in heart failure are scarce, however two studies (canine and porcine) have shown that SCS improves myocardial contractility, induces reverse remodeling and protects from ventricular arrhythmias in ischemic heart failure (Liu et al., 2012; Lopshire et al., 2009). Hence, preclinical studies on mainly ischemic heart disease models suggest a direct sym- 


\section{A: SCS90T1-4}
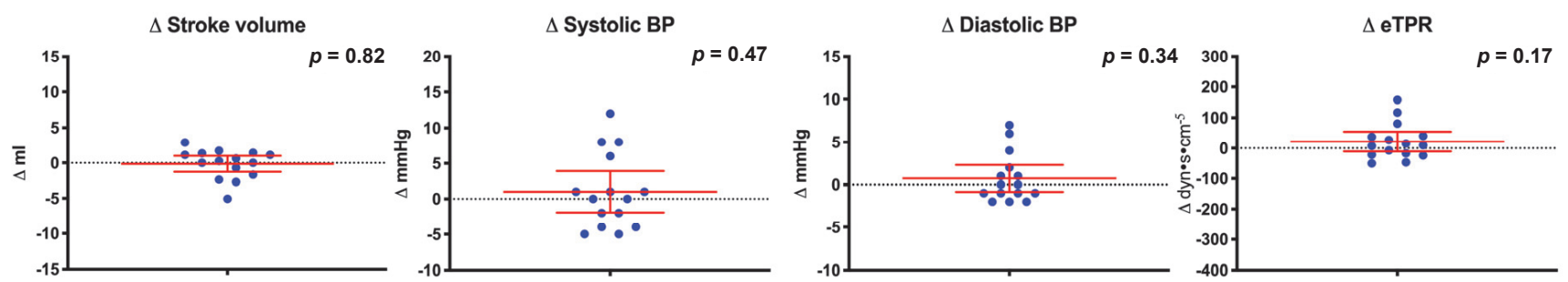

\section{B: SCS60T1-4}
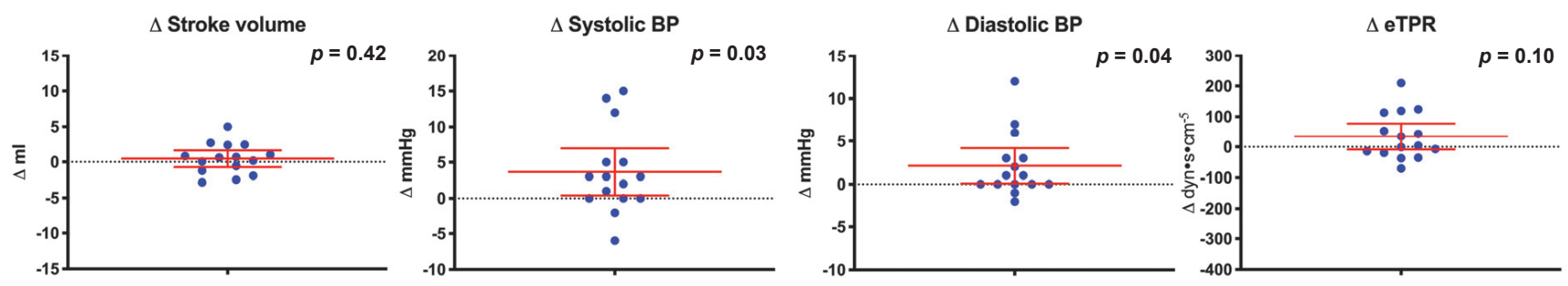

\section{C: SCS90CR}
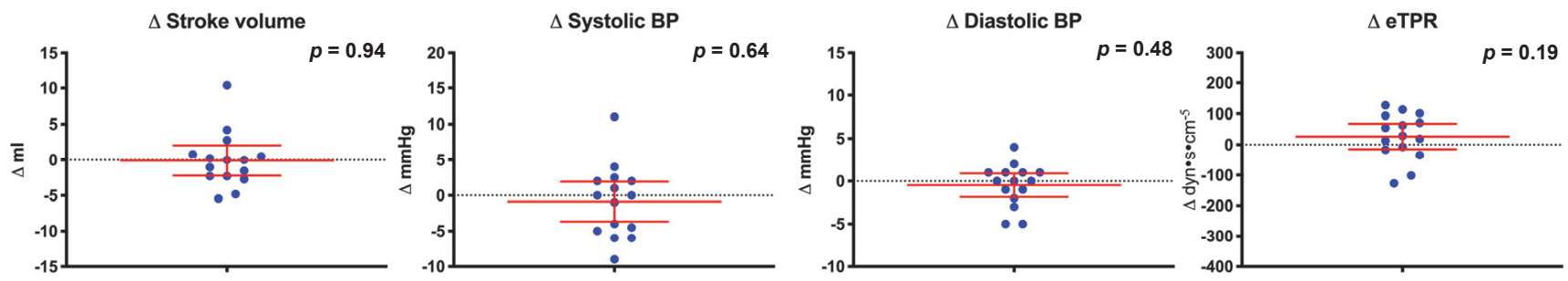

\section{D: SCS90CA}
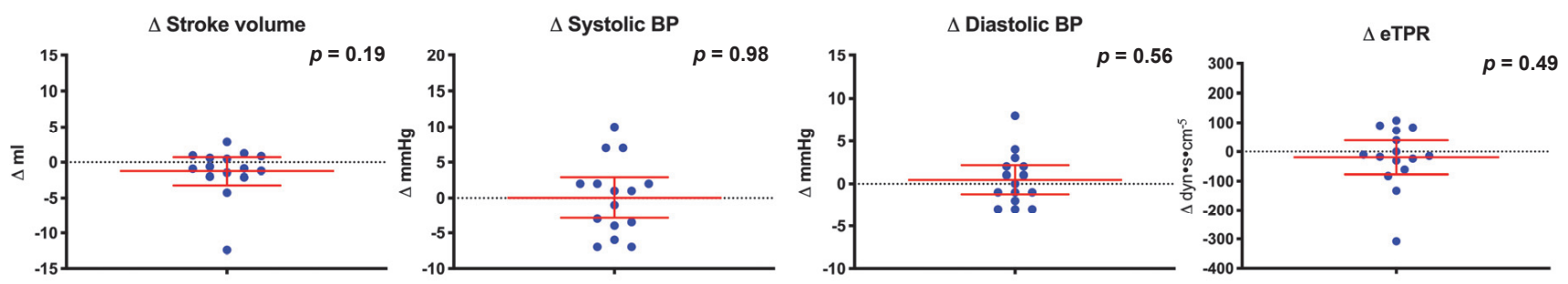

Fig. 6. Individual and mean changes of hemodynamic parameters in four spinal cord stimulation settings. Data are shown as change of mean with $95 \%$ confidence interval. BP - blood pressure; eTPR - estimated total peripheral resistance. 
patholytic effect of SCS that translates into salutary hemodynamic, structural and electrophysiological effects. Although these preclinical studies are suggestive of a salutary SCS effect in heart failure, data from clinical studies are scarce and inconsistent. The only randomized and controlled trial in patients with heart failure (DEFEAT-HF) failed to show any effect of SCS on left ventricular reverse remodeling or patient functional capacity (Zipes et al., 2016). Furthermore, in a subset of patients from the DEFEAT-HF study, we were unable to detect any mid-term effect of SCS on cardiac sympathetic nerve activity assessed by ${ }^{123}$ I-metaiodobenzylguanidine (MIBG) scintigraphy (Naar et al., 2017a). Furthermore, we did not see any beneficial effect of SCS on heart failure-associated biomarkers, i.e. renin-angiotensin-aldosterone hormonal axis activation, levels of circulating inflammation markers or norepinephrine level (Naar et al., 2017b). The main finding of the present study supports the neutral results from the DEFEAT-HF trial and previously published data on sub-groups from the main trial, as decreased sympathetic nerve activity is a fundamental presumption regarding the mechanism of action of SCS in heart failure (Linderoth and Foreman, 1999). These neutral data from the DEFEAT-HF study are in contrast to an uncontrolled observational study of SCS on patients with heart failure that demonstrated improved NYHA class, exercise capacity and left ventricular function associated with SCS (Tse et al., 2015). The reason for this is not clear but may be due to differences in patient selection, study design and therapy delivery aimed at a higher transfer of electric charge per unit time by using double leads and 24-hour SCS.

\section{Role of the baseline autonomic nervous system function on the effect of SCS in heart failure}

Clearly, most clinical data on SCS in heart failure are neutral. However, the subgroup analysis in the present study based on baseline SDNN suggests that patients with low baseline HRV may profit from SCS therapy in terms of HRV improvement. This finding is in agreement with a previous study from our group, where baseline cardiac sympathetic nerve activity assessed by ${ }^{123}$ I-MIBG scintigraphy significantly and inversely correlated with an SCS-associated change in cardiac sympathetic activity (Naar et al., 2017a). Hence, these studies imply that SCS elicits a sympatholytic or HRV-improving effect in heart failure only in patients with more pronounced sympathetic overactivity or lower baseline HRV, respectively. Therefore, our data suggest that baseline sympathetic nerve activity or autonomic balance may play a key role when examining the effect of SCS in heart failure, and suggest that the amendable substrate (level of sympathetic activity) may be important in patient selection for SCS.

\section{SCS therapy delivery}

Most preclinical and clinical studies of SCS in cardiac disease have applied near motor threshold amplitudes (animal studies) or maximal tolerated stimulation amplitude (humans) in the T1-T5 spinal cord segments, corresponding to $90 \%$ of the maximal tolerated amplitude in the T1-T4 setting used in this study. However, one experimental study in a canine ischemic heart failure model suggested that targeted spinal cord segments and stimulation amplitude may affect SCS outcome (Lopshire et al., 2009). Particularly relevant to the present study, it was demonstrated that targeting the T4 segment with $90 \%$ and $60 \%$ of motor threshold significantly reduced the heart rate, while targeting the $\mathrm{T} 1$ or $\mathrm{T} 8$ spinal cord segments did not. In the present study on heart failure patients, targeting the T4 spinal cord segment using $90 \%$ of maximal tolerated stimulation amplitude did not confer an acute effect on heart rate or HRV and neither did targeting more caudal or cranial spinal cord segments. The failure to translate the efficacy of SCS therapy from an animal heart failure model to heart failure patients could be explained by the relatively higher stimulation output used in animals: $90 \%$ of motor threshold in canines versus $90 \%$ of maximal tolerable output in humans. Preclinical studies have also only used ischemic models of heart failure with a shorter duration than clinical cases. Furthermore, differences in stimulation parameters, stimulation duration, number of leads and lead position are present among studies.

\section{Study limitations}

We acknowledge that the sample size of the present study is small and no a priori formal power calculation was performed. Nevertheless, clinical studies with similar sample sizes have been able to prove significant amelioration of HRV with SCS in patients with refractory angina pectoris (Anselmino et al., 2009; Moore et al., 2004). This is also the first report on the acute effects of SCS on HRV in patients with heart failure. The exact washout period of the effect of SCS on the autonomic nervous system is unknown and we arbitrarily chose a 35-minute period. Therefore, carry-over effects may have influenced the results in this study. Additionally, the interpretation of HRV analyses is difficult. Recent evidence implies that the nature of frequency bands from HRV spectral analysis is complex and associating a particular frequency component with divisions of the autonomic nervous system (sympathetic or parasympathetic) is too simplistic (Hayano and Yuda, 2019). Thus the interpretation of LF and LF/HF is especially challenging. Taking these limitations into account, further studies evaluating directly the sympathetic nervous system in heart failure patients treated with neuromodulation therapy would be worthwhile, for example by assessment of muscle sympathetic nerve activity (MSNA) using microneurography of the common peroneal nerve, which is standard for the direct evaluation of sympathetic nerve activity in humans, although this method does not selectively assess cardiac sympathetic nerve activity.

\section{Conclusions}

Spinal cord stimulation delivered at $60-90 \%$ of maximal tolerated output targeting the T1-T4 segments of the spinal cord does not acutely improve heart rate variability or baroreceptor sensitivity in unselected patients with heart failure, but it may improve HRV in patients with low SDNN. This finding suggests that baseline autonomic function may influence SCS response in this patient population.

\section{Acknowledgements}

The authors are grateful to Martin Mudroch and Jan Šorf for their technical assistance during device reprogramming.

\section{Conflict of interests}

The authors Jan Naar, Petr Neužil, Petr Doškář, Filip Málek, Bengt Linderoth and Göran Lind declare that they have no conflict of interests. Deborah Jaye is an employee of Medtronic, Plc. Marcus Ståhlberg has received speakers' honorarium from Medtronic, Plc. 


\section{Funding}

This work was supported by the Ministry of Health, Czech Republic, conceptual development of research organization $(\mathrm{NHH}$, 00023884), Stockholm County Council (Project\#: 108105) and an institutional research grant from Medtronic, Plc.

\section{References}

Agostoni P, Cattadori G (2009). Noninvasive cardiac output measurement: A new tool in heart failure. Cardiology 114(4): 244-246. DOI: 10.1159/000232406.

Anselmino M, Ravera L, De Luca A, Capriolo M, Bordese R, Trevi GP, Grimaldi R (2009). Spinal cord stimulation and 30-minute heart rate variability in refractory angina patients. Pacing Clin Electrophysiol 32(1): 37-42. DOI: 10.1111/j.15408159.2009.02174.x.

Azevedo ER, Parker JD (1999). Parasympathetic control of cardiac sympathetic activity: normal ventricular function versus congestive heart failure. Circulation 100(3): 274-279. DOI: 10.1161/01.cir.100.3.274.

Bernstein SA, Wong B, Vasquez C, Rosenberg SP, Rooke R, Kuznekoff LM, et al. (2012). Spinal cord stimulation protects against atrial fibrillation induced by tachypacing. Heart Rhythm 9(9): 1426-1433.e3.

Cardinal R, Ardell JL, Linderoth B, Vermeulen M, Foreman RD, Armour JA (2004). Spinal cord activation differentially modulates ischaemic electrical responses to different stressors in canine ventricles. Auton Neurosci 111(1): 37-47. DOI: 10.1016/j. autneu.2004.02.005.

Foreman RD, Linderoth B, Ardell JL, Barron KW, Chandler MJ, Hull SS, Jr., et al. (2000). Modulation of intrinsic cardiac neurons by spinal cord stimulation: implications for its therapeutic use in angina pectoris. Cardiovasc Res 47(2): 367-375. DOI: 10.1016/ s0008-6363(00)00095-x.

Goldstein DS, Bentho O, Park M-Y, Sharabi Y (2011). Low-frequency power of heart rate variability is not a measure of cardiac sympathetic tone but may be a measure of modulation of cardiac autonomic outflows by baroreflexes. Exp Physiol 96(12): 12551261. DOI: 10.1113/expphysiol.2010.056259.

Hadase M, Azuma A, Zen K, Asada S, Kawasaki T, Kamitani T, et al. (2004). Very low frequency power of heart rate variability is a powerful predictor of clinical prognosis in patients with congestive heart failure. Circ J 68(4): 343-347. DOI: 10.1253/circj.68.343.

Hayano J, Yuda E (2019). Pitfalls of assessment of autonomic function by heart rate variability. J Physiol Anthropol 38(1): 3. DOI: 10.1186/s40101-019-0193-2.

Heart rate variability: standards of measurement, physiological interpretation, and clinical use. Task Force of the European Society of Cardiology and the North American Society of Pacing and Electrophysiology (1996). Eur Heart J 17: 354-381.

Issa ZF, Zhou X, Ujhelyi MR, Rosenberger J, Bhakta D, Groh WJ, et al. (2005). Thoracic spinal cord stimulation reduces the risk of ischemic ventricular arrhythmias in a postinfarction heart failure canine model. Circulation 111(24): 3217-3720. DOI: 10.1161/ CIRCULATIONAHA.104.507897.

Jacobson AF, Senior R, Cerqueira MD, Wong ND, Thomas GS, Lopez VA, et al. (2010). Myocardial iodine-123 metaiodobenzylguanidine imaging and cardiac events in heart failure. Results of the prospective ADMIRE-HF (AdreView Myocardial Imaging for Risk Evaluation in Heart Failure) study. J Am Coll Cardiol 55(20): 2212-2221. DOI: 10.1016/j.jacc.2010.01.014.

La Rovere MT, Pinna GD, Raczak G (2008). Baroreflex sensitivity: measurement and clinical implications. Ann Noninvasive Electrocardiol 13(2): 191-207. DOI: 10.1111/j.1542474X.2008.00219.x.

Linderoth B, Foreman RD (1999). Physiology of spinal cord stimulation: review and update. Neuromodulation 2(3): 150-164. DOI: 10.1046/j.1525-1403.1999.00150.x.

Liu Y, Yue W-S, Liao S-Y, Zhang Y, Au K-W, Shuto C, et al. (2012). Thoracic spinal cord stimulation improves cardiac contractile function and myocardial oxygen consumption in a porcine model of ischemic heart failure. J Cardiovasc Electrophysiol 23(5): 534-540. DOI: 10.1111/j.1540-8167.2011.02230.x.
Lopshire JC, Zipes DP (2014). Spinal cord stimulation for heart failure: preclinical studies to determine optimal stimulation parameters for clinical efficacy. J Cardiovasc Transl Res 7(3): 321-329. DOI: 10.1007/s12265-014-9547-7.

Lopshire JC, Zhou X, Dusa C, Ueyama T, Rosenberger J, Courtney N, et al. (2009). Spinal cord stimulation improves ventricular function and reduces ventricular arrhythmias in a canine postinfarction heart failure model. Circulation 120(4): 286-294. DOI: 10.1161/CIRCULATIONAHA.108.812412.

Moore R, Groves D, Nolan J, Scutt D, Pumprla J, Chester MR (2004). Altered short term heart rate variability with spinal cord stimulation in chronic refractory angina: evidence for the presence of procedure related cardiac sympathetic blockade. Heart 90(2): 211-212. DOI: 10.1136/hrt.2002.002998.

Mortara A, La Rovere MT, Pinna GD, Prpa A, Maestri R, Febo O, et al. (1997). Arterial baroreflex modulation of heart rate in chronic heart failure: clinical and hemodynamic correlates and prognostic implications. Circulation 96(10): 3450-3458. DOI: 10.1161/01.cir.96.10.3450.

Naar J, Jaye D, Linde C, Neužil P, Doškář P, Málek F, et al. (2017a). Effects of Spinal Cord Stimulation on Cardiac Sympathetic Nerve Activity in Patients with Heart Failure. Pacing Clin Electrophysiol 40(5): 504-513. DOI: 10.1111/pace.13050.

Naar J, Jaye D, Linde C, Neužil P, Doškář P, Málek F, et al. (2017b). Spinal cord stimulation in heart failure: effect on diseaseassociated biomarkers. Eur J Heart Fail 19(2): 283-286. DOI: $10.1002 /$ ejhf.702.

Nakata T, Nakajima K, Yamashina S, Yamada T, Momose M, Kasama S, et al. (2013). A pooled analysis of multicenter cohort studies of (123) I-mIBG imaging of sympathetic innervation for assessment of long-term prognosis in heart failure. JACC Cardiovasc Imaging 6(7): 772-784. DOI: 10.1016/j. jcmg.2013.02.007.

Odenstedt J, Linderoth B, Bergfeldt L, Ekre O, Grip L, Mannheimer C, Andrell P (2014). Spinal cord stimulation effects on myocardial ischemia, infarct size, ventricular arrhythmia, and noninvasive electrophysiology in a porcine ischemiareperfusion model. Heart Rhythm 8(6): 892-898. DOI: 10.1016/j. hrthm.2011.01.029.

Osterziel KJ, Hänlein D, Willenbrock R, Eichhorn C, Luft F, Dietz R (1995). Baroreflex sensitivity and cardiovascular mortality in patients with mild to moderate heart failure. Br Heart J 73(6): 517-522. DOI: 10.1136/hrt.73.6.517.

Packer M (1992). The neurohormonal hypothesis: a theory to explain the mechanism of disease progression in heart failure. J Am Coll Cardiol 20(1): 248-254. DOI: 10.1016/0735-1097(92)90167-1.

Parlow J, Viale JP, Annat G, Hughson R, Quintin L (1995). Spontaneous cardiac baroreflex in humans. Comparison with drug-induced responses. Hypertension 25(5): 1058-1068. DOI: 10.1161/01.hyp.25.5.1058.

Rahman F, Pechnik S, Gross D, Sewell L, Goldstein DS (2011). Low frequency power of heart rate variability reflects baroreflex function, not cardiac sympathetic innervation. Clin Auton Res 21(3): 133-141. DOI: 10.1007/s10286-010-0098-y.

Simpson EL, Duenas A, Holmes MW, Papaioannou D, Chilcott J (2009). Spinal cord stimulation for chronic pain of neuropathic or ischaemic origin: systematic review and economic evaluation. Health Technol Assess 13(17): iii, ix-x, 1-154. DOI: 10.3310/ hta13170.

Tse H-F, Turner S, Sanders P, Okuyama Y, Fujiu K, Cheung C-W, et al. (2015). Thoracic Spinal Cord Stimulation for Heart Failure as a Restorative Treatment (SCS HEART study): first-in-man experience. Heart Rhythm 12(3): 588-595. DOI: 10.1016/j. hrthm.2014.12.014.

Wang W, Chen JS, Zucker IH (1990). Carotid sinus baroreceptor sensitivity in experimental heart failure. Circulation 81(6): 1959-1966. DOI: 10.1161/01.cir.81.6.1959.

Wang Y-P, Cheng Y-J, Huang C-L (2004). Spontaneous baroreflex measurement in the assessment of cardiac vagal control. Clin Auton Res 14(3): 189-193. DOI: 10.1007/s10286-004-0192-0.

Zipes DP, Neuzil P, Theres H, Caraway D, Mann DL, Mannheimer C, et al. (2016). Determining the Feasibility of Spinal Cord Neuromodulation for the Treatment of Chronic Systolic Heart Failure: The DEFEAT-HF Study. JACC Heart Fail 4(2): 129-136. DOI: 10.1016/j.jchf.2015.10.006. 